\title{
Phase-resolved X-ray and optical spectroscopy of the massive binary HD 93403*
}

\author{
G. Rauw ${ }^{1}$, J.-M. Vreux ${ }^{1}$, I. R. Stevens ${ }^{2,1}$, E. Gosset ${ }^{1, \star \star}$, H. Sana ${ }^{1, \star \star \star}$, C. Jamar ${ }^{3}$, and K. O. Mason ${ }^{4}$ \\ 1 Institut d'Astrophysique, Université de Liège, Allée du 6 Août, Bât B5c, 4000 Liège (Sart Tilman), Belgium \\ 2 School of Physics \& Astronomy, University of Birmingham, Edgbaston Birmingham B15 2TT, UK \\ 3 Centre Spatial de Liège, Université de Liège, Avenue du Pré-Aily, 4031 Angleur, Belgium \\ 4 Mullard Space Science Laboratory, University College London, Holmbury St. Mary, Dorking, Surrey, \\ RH5 6NT, UK
}

Received 24 July 2001 / Accepted 3 April 2002

\begin{abstract}
We report the first results of a campaign aimed at the study of early-type binaries with the $X M M$ Newton observatory. Phase-resolved EPIC spectroscopy of the eccentric binary HD 93403 reveals a clear orbital modulation of the X-ray luminosity as a function of the orbital phase. Below $1.0 \mathrm{keV}$, the observed X-ray flux is modulated by the opacity of the primary wind. Above $1.0 \mathrm{keV}$, the observed variation of the X-ray flux is roughly consistent with a $1 / r$ dependence expected for an adiabatic colliding wind interaction. HD 93403 appears less overluminuous in X-rays than previously thought and a significant fraction of the total X-ray emission arises probably within the winds of the individual components of the binary. Optical monitoring of the system reveals strong variability of the He II $\lambda 4686$ and $\mathrm{H} \alpha$ line profiles. The He II $\lambda 4686$ line displays a broad asymmetrical emission component which is found to be significantly stronger between phases 0.80 and 0.15 than around apastron. This suggests that part of the emission arises in the interaction region and most probably in the trailing arm of a shock cone wrapped around the secondary. Some absorption lines of the secondary's spectrum display equivalent width variations reminiscent of the so-called Struve-Sahade effect. The differences in behaviour between individual lines suggest that the temperature may not be the only relevant parameter that controls this effect.
\end{abstract}

Key words. stars: early-type - binaries: spectroscopic - stars: individual: HD 93403 - stars: winds, outflows X-rays: stars

\section{Introduction}

Early-type binaries, consisting either of a Wolf-Rayet (WR) star with an O-star companion or of two O-stars, can be the scene of a strong interaction between the stellar winds of the two components. This wind collision is expected to produce an X-ray emission that displays significant phase-locked variability either as a consequence of the changing wind opacity along the line of sight towards the shock or as a result of the changing orbital separation in eccentric binaries (see e.g. Stevens et al. 1992; Pittard \& Stevens 1997). A comparison of the observed spectra and light curves with predictions from numerical simulations

\footnotetext{
Send offprint requests to: G. Rauw,

e-mail: rauw@astro.ulg.ac.be

* Based on observations with XMM-Newton, an ESA Science Mission with instruments and contributions directly funded by ESA Member states and the USA (NASA). Also based on observations collected at the European Southern Observatory (La Silla, Chile).

$\star \star$ Research Associate FNRS (Belgium).

$\star \star \star$ Research Fellow FNRS (Belgium).
}

should help us to better understand the hydrodynamics of stellar winds.

While a number of WR $+\mathrm{O}$ binaries have been extensively monitored with previous and current X-ray satellites (for a recent review see e.g. van der Hucht 2002), so far only a few colliding wind $\mathrm{O}+\mathrm{O}$ binaries have been studied in detail in the X-ray domain. Corcoran (1996) presented the ROSAT-PSPC light curves of sixteen O-type binaries (not including HD 93403, the system discussed in this paper) and found evidence for a phase-locked modulation in at least four cases. Pittard et al. (2000) analyzed two $A S C A$ observations of the highly eccentric system $\iota$ Ori obtained near periastron and apastron. Strong differences between these phases were a priori expected, but the observations proved otherwise: both the luminosity and the spectral shapes were found to be remarkably similar. This suggests that the bulk of the X-ray emission in $\iota$ Ori is intrinsic to one of the components rather than being associated with the wind interaction.

The signatures of wind interaction phenomena in $\mathrm{O}+\mathrm{O}$ binaries may also be found in their optical spectra. Indeed, phase-locked variability of the profiles of optical 
Table 1. Journal of the XMM-Newton observations of HD 93403. The first column lists the spacecraft revolution number, the second and third columns provide the date and the corresponding orbital phase of HD 93403 relative to periastron passage (according to the ephemeris of Rauw et al. 2000) at mid-exposure for the EPIC-MOS instruments. Note that the pn exposures started about $4000 \mathrm{~s}$ later than the MOS observations. The following six columns list the effective exposure time (in seconds) and the background corrected count rate $\left(\mathrm{cts} \mathrm{s}^{-1}\right)$ of $\mathrm{HD} 93403$ for energies above $0.5 \mathrm{keV}$. The last two columns provide the relative orbital separation between the components (in units of the semi-major axis) and the position angle (PA) of the binary axis. A PA of $0^{\circ}$ corresponds to the O5.5 I primary star being "in front".

\begin{tabular}{|c|c|c|c|c|c|c|c|c|c|c|}
\hline \multirow[t]{2}{*}{ Rev. } & \multirow{2}{*}{$\begin{array}{c}\text { JD } \\
-2450000.0\end{array}$} & \multirow[t]{2}{*}{$\Phi$} & \multicolumn{2}{|r|}{ MOS1 } & \multicolumn{2}{|r|}{ MOS2 } & \multicolumn{2}{|r|}{ pn } & \multirow[t]{2}{*}{$r / a$} & \multirow{2}{*}{$\begin{array}{l}\text { PA } \\
\left({ }^{\circ}\right)\end{array}$} \\
\hline & & & Exp. & Count rate & Exp. & Count rate & Exp. & Count rate & & \\
\hline 191 & 1902.995 & 0.265 & 8865 & $0.208 \pm 0.005$ & 8869 & $0.213 \pm 0.005$ & 4472 & $0.466 \pm 0.011$ & 1.060 & 127 \\
\hline 193 & 1906.815 & 0.518 & 8666 & $0.191 \pm 0.005$ & 8670 & $0.186 \pm 0.005$ & 4344 & $0.419 \pm 0.011$ & 1.233 & 63 \\
\hline 195 & 1910.234 & 0.745 & 8763 & $0.199 \pm 0.005$ & 8766 & $0.199 \pm 0.005$ & 4416 & $0.405 \pm 0.011$ & 1.073 & 5 \\
\hline 212 & 1944.435 & 0.011 & 11492 & $0.228 \pm 0.005$ & 11491 & $0.220 \pm 0.005$ & 6871 & $0.487 \pm 0.009$ & 0.767 & 119 \\
\hline
\end{tabular}

emission lines such as $\mathrm{H} \alpha$ or He II $\lambda 4686$ provides a powerful diagnostic that can be used to constrain the geometry of the interaction regions in these systems (e.g. Thaller et al. 2001; Rauw et al. 1999, 2001; Sana et al. 2001).

In this paper, we present the results of our XMMNewton observing campaign of the eccentric massive binary HD 93403 (O5.5 I + O7 V, $P_{\text {orb }}=15.093$ days, $e=$ 0.234, Rauw et al. 2000) in the Carina complex. HD 93403 is an ideal system to investigate the effects of the eccentricity on the X-ray emission generated in a colliding wind process. In fact, because of the probably rather low orbital inclination $\left(i \simeq 30^{\circ}\right.$, Rauw et al. 2000), we expect little variability of the circumstellar column density towards the shock and any phase dependence of the X-ray emission therefore most probably reflects the changing separation between the two stars.

EINSTEIN observations of HD 93403 revealed an exceptionally large X-ray excess luminosity above the "canonical" $L_{\mathrm{X}} / L_{\mathrm{bol}}$ relation for single O-stars, making HD 93403 one of the most extreme $\mathrm{O}+\mathrm{O}$ binary systems in the sample of Chlebowski \& Garmany (1991). In our present analysis, we derive however a significantly lower value for $L_{\mathrm{X}} / L_{\mathrm{bol}}$ (see Sect. 3.3).

In the following, we focus on the EPIC medium resolution spectra of HD 93403. We discuss the X-ray light curve in conjunction with the results of a ground-based campaign that revealed variability in the optical spectrum of HD 93403. Some preliminary results of the optical monitoring were already presented by Rauw et al. (2002a).

\section{Observations and data reduction}

\subsection{XMM-Newton observations}

Four phase-constrained observations of HD 93403 were obtained with the XMM-Newton observatory (Jansen et al. 2001) as part of the guaranteed time observation programme of the XMM Optical Monitor consortium (Mason et al. 2001). The journal of observations is given in Table 1. The first three pointings were scheduled during the same orbital cycle of HD 93403, while the last pointing was obtained two cycles later. The two EPIC MOS instruments were operated in the full frame mode (Turner et al. 2001) whilst the EPIC-pn camera was used in the extended full frame mode (Strüder et al. 2001). All three EPIC instruments used the thick filter to reject optical and UV light.

We used the XMM-Newton Science Analysis System (SAs, version 5.0.1) to process the raw EPIC data. For the MOS (resp. pn) observations only events with pattern 0-12 (resp. 0) were considered (Turner et al. 2001). We checked that the data were not affected by any strong background flares. No indication of pile-up was found in our data. The spectrum of HD 93403 was accumulated over a radius of $60^{\prime \prime}$ and the background spectrum was obtained over an annulus around the source region (outer radius $\left.85^{\prime \prime}\right)$. For the pn detector, we truncated the extraction region to include only events from CCD 1 . We adopted the redistribution matrices provided by the EPIC instrument teams (versions available in May 2001). We used the sAS to build the appropriate ancillary response file for each observation. The spectra were binned to reach a minimum of 25 counts per channel and the background corrected spectra were analyzed using the XSPEC software (version 11.00).

A preliminary discussion of the X-ray sources in the EPIC field of view around HD 93403 can be found in Rauw et al. (2002b).

\subsection{Optical spectra}

In the present paper, we use a set of sixteen echelle spectra of HD 93403 obtained with the Fiber-fed Extended Range Optical Spectrograph (FEROS, Kaufer et al. 1999) attached to the ESO $1.52 \mathrm{~m}$ telescope at La Silla. The data were collected during four observing runs in AprilMay 1999, May 1999, 2000 and 2001 (the whole dataset being spread over 49 orbital cycles). The observations and reductions of the first three observing runs (13 spectra) are described in Rauw et al. (2000). In May 2001, three additional spectra were obtained on HJD 2452037.607, 2452038.571 and 2452040.554 corresponding to phases $\Phi=0.184,0.248$ and 0.379 respectively. The latter data were reduced with a modified version of the FEROS context working under the MIDAS environment. The MIDAS routines were adapted following recommendations by Dr. $\mathrm{H}$. Hensberge in order to optimize the blaze correction. 


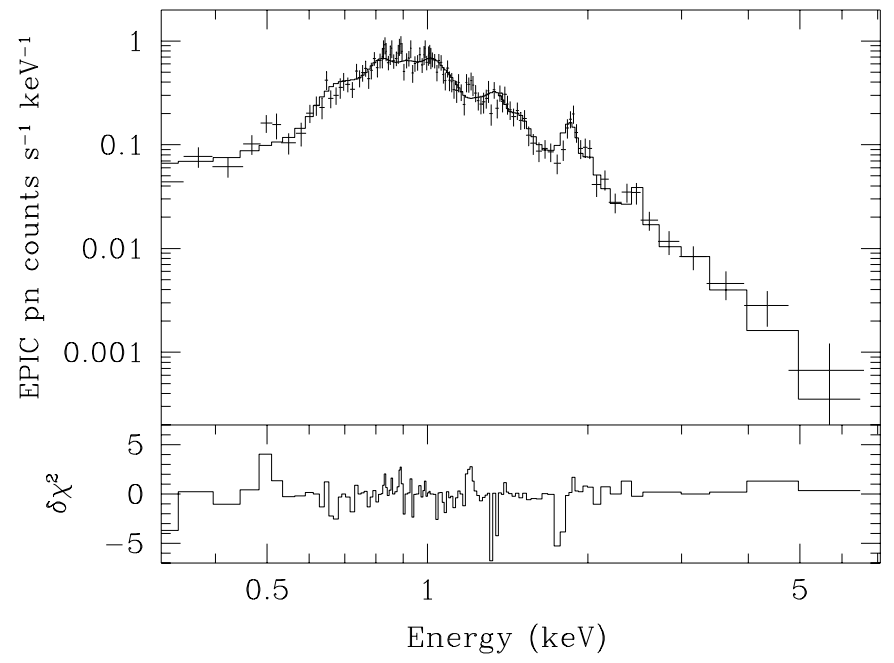

Fig. 1. EPIC pn spectrum of HD 93403 at $\Phi=0.011$ and bestfitting 2-T MEKAL model (from Table 2). The lower panel yields the contribution to the $\chi^{2}$ per energy bin with the sign of the $(\mathrm{O}-\mathrm{C})$ residual.

\section{The X-ray data}

\subsection{Spectral fits}

To quantify the properties of the X-ray emission from HD 93403, we fitted the EPIC spectra with a variety of simple models. The spectral fits were restricted to energies above $0.5 \mathrm{keV}$. This analysis is only meant to characterize the overall spectral shape and its variability as a function of orbital phase. The models used here are certainly too simplistic to accurately describe the physical conditions in the emitting plasma and the best fit parameters should therefore not be overinterpreted. The most evident flaw of this simple spectral modelling is that the cross sections of the absorbing column do not take into account the ionization of the wind material ${ }^{1}$. What prevents us from applying a proper wind absorption model such as the one of Waldron et al. (1998) is that in a colliding wind binary, like HD 93403, the geometry of the emitting and absorbing regions is far more complex than in a single star. An accurate modelling of this situation requires the use of sophisticated 3-D hydrodynamic models (e.g. Pittard 2000). This is beyond the scope of the present work and will be the subject of a forthcoming paper.

Since the best fits obtained for the three EPIC instruments are very similar, we focus here on the results obtained for a simultaneous fit of the data from all EPIC instruments.

First we tried to fit the EPIC spectra of HD 93403 with solar metallicity thermal plasma MEKAL models (Mewe et al. 1985; Kaastra 1992). Single temperature models do not provide a decent fit to the data $\left(\chi_{\nu} \geq 2.2\right)$. As a next step, we fitted the spectra using two temperature solar abundance MEKAL models allowing distinct column

\footnotetext{
${ }^{1}$ We note however that restricting the fits to energies above $0.5 \mathrm{keV}$ should considerably reduce the impact of this assumption.
}
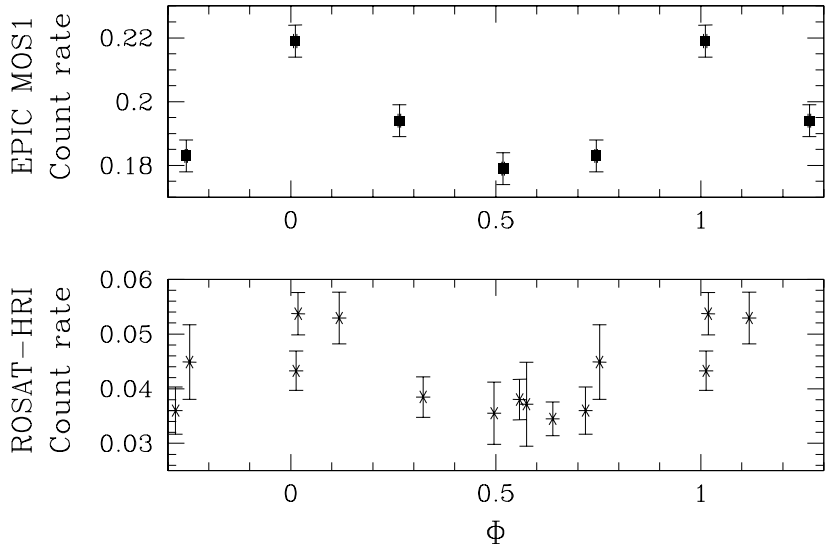

Fig. 2. X-ray light curve of HD 93403. The top panel yields the EPIC MOS1 count rate in the $0.5-2.5 \mathrm{keV}$ band. $1-\sigma$ error bars are indicated. The MOS2 and pn data display very similar variations. The lower panel illustrates the ROSAT-HRI light curve extracted from observation rh202331n00 (see text).

densities for both components, but requiring them to be larger than the interstellar Hi column density. Chlebowski \& Garmany (1991) measured the interstellar Ly $\alpha$ line as observed on IUE spectra of HD 93403 to estimate $N_{\mathrm{H}, \text { ISM }}=0.367 \times 10^{22} \mathrm{~cm}^{-2}$. For the fits in Table 2, we thus forced the individual columns of our $2-\mathrm{T}$ model to be larger than $0.37 \times 10^{22} \mathrm{~cm}^{-2}$. Apart from the X-ray fluxes, the best-fitting spectral parameters in Table 2 reveal surprisingly little variability over the orbital cycle.

Finally, we also tested a two component model consisting of a 1-T MEKAL thermal model + a power-law spectrum. The fits are of comparable quality to those obtained with the 2-T MEKAL models and yield $k T \simeq 0.60 \mathrm{keV}$ and a photon index $\Gamma \geq 3$.0. Theoretical models predict that strong shocks in stellar winds, either due to intrinsic instabilities (Chen \& White 1991) or to colliding wind interactions in binaries (Eichler \& Usov 1993), can accelerate free electrons to relativistic energies through the first-order Fermi mechanism. This process is expected to account for the non-thermal radio emission observed in a subset of early-type stars ${ }^{2}$. Given the enormous supply of photospheric UV photons in the winds of O-stars, inverseCompton scattering by relativistic electrons is expected to generate a non-thermal X-ray emission. However, the bestfitting photon indices we derive are much steeper than the values expected from theoretical models of the first order Fermi mechanism $(\Gamma \simeq 1.5$, Chen \& White 1991). The existence of a non-thermal component in the X-ray spectrum of HD 93403 is therefore unlikely.

\subsection{Phase-locked variability}

The fits of the EPIC spectra indicate surprisingly little variability of the spectral shape as a function of orbital phase. However, they do reveal a phase-dependence of the observed X-ray flux. In fact, the fluxes given in Table 2 and the count rates plotted in Fig. 2 indicate that

\footnotetext{
2 No detection of a radio emission (be it thermal or nonthermal) from HD 93403 is reported in the literature.
} 
Table 2. Best fitting parameters of the solar metallicity 2-T MEKAL fits (wabs ISM $*\left(\right.$ wabs $_{1} * \operatorname{mekal}_{1}+$ wabs $_{2} *$ mekal $_{2}$ )) to the EPIC spectra of HD 93403. The quoted column densities do not include the interstellar column set to $0.37 \times 10^{22} \mathrm{~cm}^{-2}$. The normalization coefficients correspond to $\frac{10^{-14}}{4 \pi d^{2}} \int n_{\mathrm{e}} n_{\mathrm{H}} \mathrm{d} V$, where $d, n_{\mathrm{e}}$ and $n_{\mathrm{H}}$ are respectively the distance of HD 93403 (in cm), the electron density and the hydrogen density $\left(\right.$ in $\mathrm{cm}^{-3}$ ) in the X-ray emitting plasma. Error bars correspond to the 90 per cent confidence ranges. $f_{\mathrm{X}}^{\mathrm{a}}$ and $f_{\mathrm{X}}^{\mathrm{b}}$ yield the observed (i.e. absorbed) fluxes (in $10^{-12} \mathrm{erg} \mathrm{cm}^{-2} \mathrm{~s}^{-1}$ ) integrated over the energy bands $0.5-2.5 \mathrm{keV}$ and $0.5-10.0 \mathrm{keV}$ respectively.

\begin{tabular}{|c|c|c|c|c|c|c|c|c|c|c|}
\hline$\Phi$ & $\begin{array}{c}N_{\mathrm{H}, 1} \\
\left(10^{22} \mathrm{~cm}^{-2}\right) \\
\end{array}$ & $\begin{array}{c}k T_{1} \\
(\mathrm{keV})\end{array}$ & norm $_{1}$ & $\begin{array}{c}N_{\mathrm{H}, 2} \\
\left(10^{22} \mathrm{~cm}^{-2}\right) \\
\end{array}$ & $\begin{array}{c}k T_{2} \\
(\mathrm{keV})\end{array}$ & norm $_{2}$ & $\chi_{\nu}^{2}$ & dof & $f_{\mathrm{X}}^{\mathrm{a}}$ & $f_{\mathrm{X}}^{\mathrm{b}}$ \\
\hline 0.265 & $0.45_{-.10}^{+.08}$ & $0.24_{-.02}^{+.03}$ & $1.30 \times 10^{-2}$ & $0.88_{-.24}^{+.36}$ & $1.25_{-.09}^{+.10}$ & $1.55 \times 10^{-3}$ & 1.13 & 191 & 1.14 & 1.36 \\
\hline 0.518 & $0.50_{-.09}^{+.09}$ & $0.21_{-.02}^{+.02}$ & $1.96 \times 10^{-2}$ & $0.85_{-.17}^{+.36}$ & $1.01_{-.06}^{+.08}$ & $1.74 \times 10^{-3}$ & 1.12 & 169 & 1.03 & 1.18 \\
\hline 0.745 & $0.51_{-.09}^{+.08}$ & $0.21_{-.02}^{+.02}$ & $1.70 \times 10^{-2}$ & $0.80_{-.12}^{+.17}$ & $1.05_{-.06}^{+.06}$ & $2.01 \times 10^{-3}$ & 1.04 & 179 & 1.07 & 1.25 \\
\hline 0.011 & $0.36_{-.10}^{+.09}$ & $0.25_{-.02}^{+.03}$ & $0.76 \times 10^{-2}$ & $0.77_{-.10}^{+.11}$ & $0.92_{-.06}^{+.04}$ & $2.18 \times 10^{-3}$ & 0.99 & 260 & 1.24 & 1.38 \\
\hline
\end{tabular}

the system appears about $20 \%$ brighter (in the $0.5-2.5 \mathrm{keV}$ band) around periastron compared to the apastron observation.

To check that this behaviour is stable on time scales that are much longer than the orbital period, we have extracted archive ROSAT observations. HD 93403 was observed at several occasions with either the PSPC or the HRI instrument. The ROSAT pointings were usually centered on $\eta$ Car. Therefore, HD 93403 lies rather close to the inner ring of the PSPC mesh and the source was sometimes shadowed by the mesh as a result of the spacecraft wobbling. Because of these difficulties we shall not consider the PSPC data any further and we will focus on the HRI exposures instead.

The background- and exposure-corrected HRI count rates were measured using the ASTERIX software. Figure 2 illustrates the light curve corresponding to observation rh202331n00 (obtained between JD 2450805.8 and JD 2450844.2). Despite the rather large error bars on individual data points the variability of the HRI count rate is significant at the $>99.5 \%$ level (fitting a constant count rate to the HRI data yields $\chi_{\nu}^{2}=2.87$ for 9 d.o.f.): HD 93403 appears brighter near $\Phi=0.0$ and fainter around $\Phi=0.5$, in fair agreement with our EPIC light curve. The overall HRI light curve (including all HRI observations) yields very similar results.

Our XMM-Newton data provide the most complete phase-resolved X-ray spectroscopy of an $\mathrm{O}+\mathrm{O}$ colliding wind system to date. In Fig. 3 we have plotted the MOS1 count rates over different energy bands as a function of phase. Also illustrated in this figure are the orbital separation and the position angle as a function of phase $\Phi$. The count rate in the medium energy band (that yields the dominant contribution to the global flux) follows the trend already seen for the entire energy range in Fig. 2. On the other side, the flux in the soft band is minimum at $\Phi=0.75$. From Table 2 and Fig. 3, we find that phase 0.75 corresponds to the time of conjunction with the O5.5 I primary, and its denser wind, being in front $\left(\mathrm{PA}=5^{\circ}\right)$. Although the orbital inclination is rather low, it seems likely that the increase of the wind column density along the line of sight at $\Phi=0.75$ is responsible for the

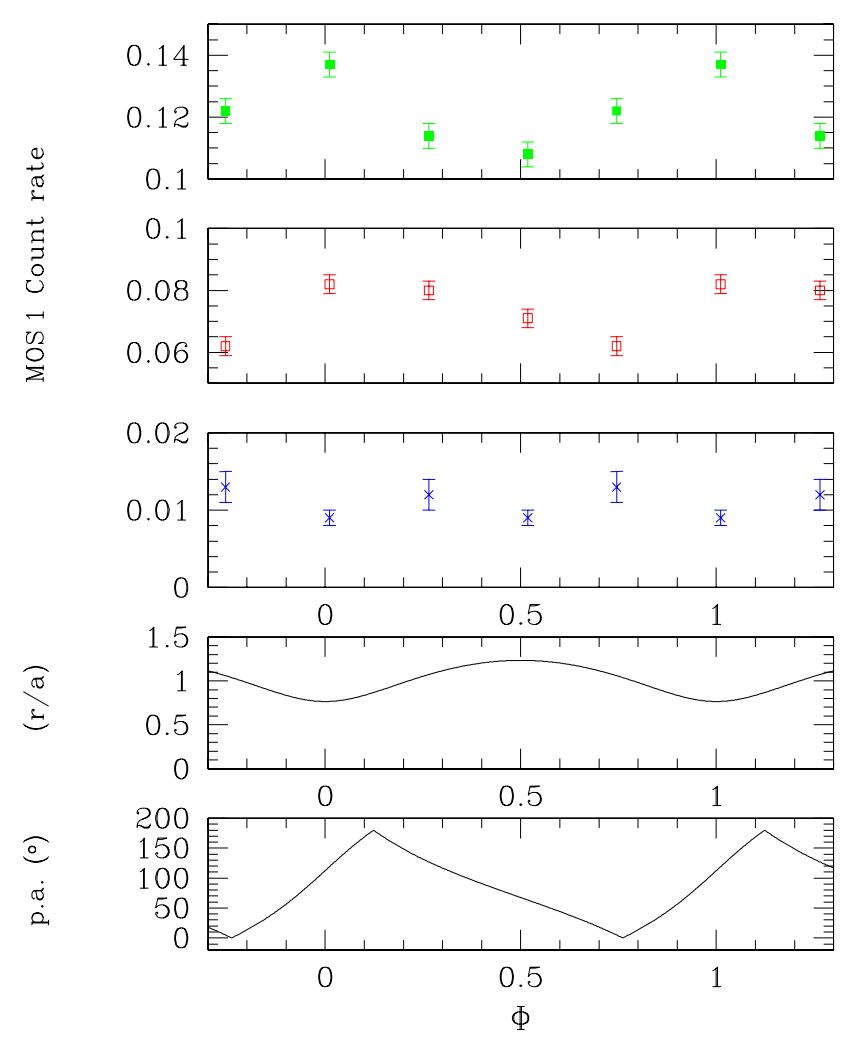

Fig. 3. X-ray light curve of HD 93403 over different energy ranges. The upper, second and third panels display the MOS1 count rates respectively over the 1.0-2.5 (medium), 0.5-1.0 (soft) and 2.5-10.0 keV (hard) energy bands. 1- $\sigma$ error bars are indicated. The MOS2 and pn data yield very similar light curves. The fourth panel yields the relative orbital separation $(r / a)$ between the components of HD 93403. Finally, the lower panel provides the position angle of the binary axis. A PA of $0^{\circ}$ corresponds to the primary star being "in front" of the secondary.

observed drop in the soft flux. Finally, the variations in the hard band are less significant (at the $80 \%$ level).

\subsection{Discussion}

As pointed out above, the EPIC spectra of HD 93403 reveal surprisingly little variability: the model temperatures $k T_{1}$ and $k T_{2}$ vary by less than $20 \%$ and $35 \%$ 
Table 3. Wind properties of the components of HD 93403 (see text for details). $\mathcal{R}$ yields the "wind momentum ratio" (e.g. Stevens et al. 1992). The last two rows provide the radii of the "instantaneous Roche lobes" at periastron and apastron.

\begin{tabular}{lcc}
\hline \hline & Primary & Secondary \\
\hline Spectral type & O5.5 I & O7 V \\
$T_{\text {eff }}(\mathrm{K})$ & 39300 & 40100 \\
$\log \left(L_{\mathrm{bol}} / L_{\odot}\right)$ & 6.02 & 5.41 \\
$\log \dot{M}\left(M_{\odot} \mathrm{yr}^{-1}\right)$ & -5.29 & -6.36 \\
$v_{\infty}\left(\mathrm{km} \mathrm{s}^{-1}\right)$ & 2600 & 2600 \\
$\mathcal{R}=\sqrt{\frac{\dot{\mathrm{M}}_{1} v_{\infty, 1}}{\dot{\mathrm{M}}_{2} v_{\infty, 2}}}$ & \multicolumn{2}{c}{3.3} \\
$R_{\mathrm{RL}} \sin i(\Phi=0.0)\left(R_{\odot}\right)$ & 20.7 & 16.0 \\
$R_{\mathrm{RL}} \sin i(\Phi=0.5)\left(R_{\odot}\right)$ & 33.3 & 25.8 \\
\hline
\end{tabular}

respectively. At first sight, this is somewhat reminiscent of the case of $\iota$ Ori (Pittard et al. 2000). ASCA observations of $\iota$ Ori (O9 III + B1 III, $e=0.764$, Marchenko et al. 2000) failed to reveal the expected variability between the periastron and apastron phases. However, in the case of HD 93403, we do observe a phase-dependence of the observed X-ray flux.

To better understand the origin of the variations at different energies we need to first consider some fundamental properties of the components of HD 93403 listed in Table 3. To estimate the mass loss rates of the components, we use the empirical formula proposed by Lamers \& Leitherer (1993). The effective temperatures and luminosities are adopted from the estimates of Rauw et al. (2000). Howarth et al. (1997) determined a terminal velocity of $2616 \mathrm{~km} \mathrm{~s}^{-1}$ for the combined stellar wind of HD 93403. Here we assume that both components have the same $v_{\infty}$ of $2600 \mathrm{~km} \mathrm{~s}^{-1}$.

High resolution $\mathrm{X}$-ray spectroscopy of single massive stars indicates that the emitting plasma is distributed out to large radii in the stellar wind. Analyses of the He-like triplets of various ions suggest that the bulk of the line emission arises from regions just outside the radius of optical depth unity for the respective wavelength (e.g. Kahn et al. 2001).

We can use the parameters in Table 3 to estimate the radius of optical depth unity in the winds of the two stars at different energies. Assuming homogeneous and spherically symmetric winds, we find that, for photons of energy $0.5 \mathrm{keV}, \tau=1$ occurs at $9 R_{*}\left(\simeq 216 R_{\odot}\right)$ and $2 R_{*}$ $\left(\simeq 20 R_{\odot}\right)$ for the primary and secondary of HD 93403 respectively. At $1.5 \mathrm{keV}$, the primary wind remains optically thick out to $47 R_{\odot}$. Comparing these figures to the orbital dimensions $\left(a \sin i=63.2 R_{\odot}\right)$, we see that even for inclinations of order $30^{\circ}$, the whole orbit lies inside the $\tau=1$ radius at $0.5 \mathrm{keV}$ except of course for the solid angle occupied by the cavity created by the secondary wind (see Fig. 4). This situation has two important consequences:

- any soft X-ray emission produced in the inner region of the wind interaction zone will be heavily absorbed by the winds

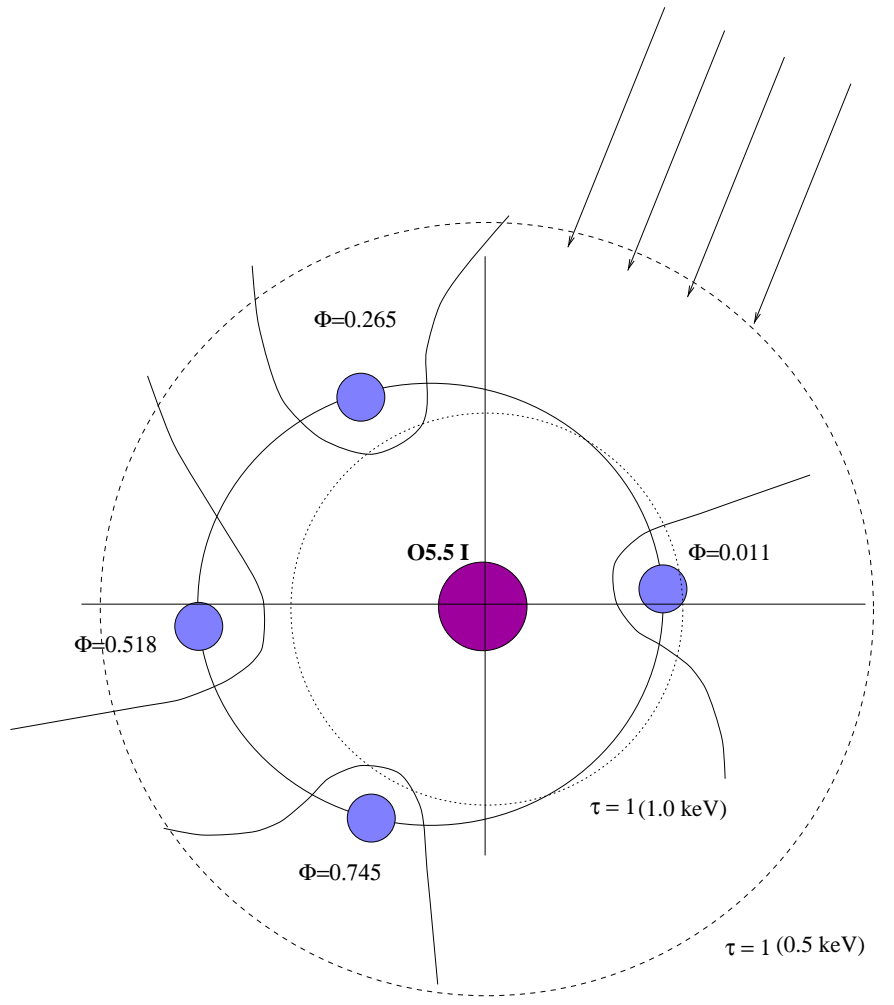

Fig. 4. Schematic view of the HD 93403 binary at the time of the four $X M M$ observations. The dimensions of the stars and the orbital separation correspond to an orbital inclination of $30^{\circ}$. The arrows indicate the direction of the observer's line of sight projected on the orbital plane. The dashed and the dotted circles correspond to the surfaces of optical depth unity for the primary wind at $0.5 \mathrm{keV}$ and $1.0 \mathrm{keV}$ respectively.

- the soft $(0.5-1.0 \mathrm{keV}) \mathrm{X}$-ray emission observed in the EPIC spectra of HD 93403 most likely arises in the outer regions of the individual stellar winds and the variability in this energy range is probably associated with pure opacity effects.

In the medium energy band (1.0-2.5 keV), optical depth effects are much smaller and should have less impact on the light curve. Figure 3 shows that at least in this energy range, the variation is roughly consistent with a $1 / r$ modulation, where $r$ stands for the instantaneous separation between the stars. Such a behaviour is indeed expected in some colliding wind binaries. In fact, if the plasma in the wind interaction zone is adiabatic, the X-ray luminosity of a wind collision is expected to scale roughly as $L_{\mathrm{X}} \propto v^{-3.2} r^{-1}$ (Stevens et al. 1992), where $v$ stands for the preshock velocity of the gas.

Referring to the cooling parameter $\chi$ introduced by Stevens et al. (1992) and the wind parameters listed in Table 3, we find that the plasma in the wind interaction zone of a system such as HD 93403 is a priori most probably adiabatic $(\chi \sim 3-5)$, except perhaps around periastron $(\chi \sim 0.4$ for the secondary wind) where radiative cooling could become effective (e.g. Pittard \& Stevens 1997). From the sole dependence of $L_{\mathrm{X}}$ on the orbital 

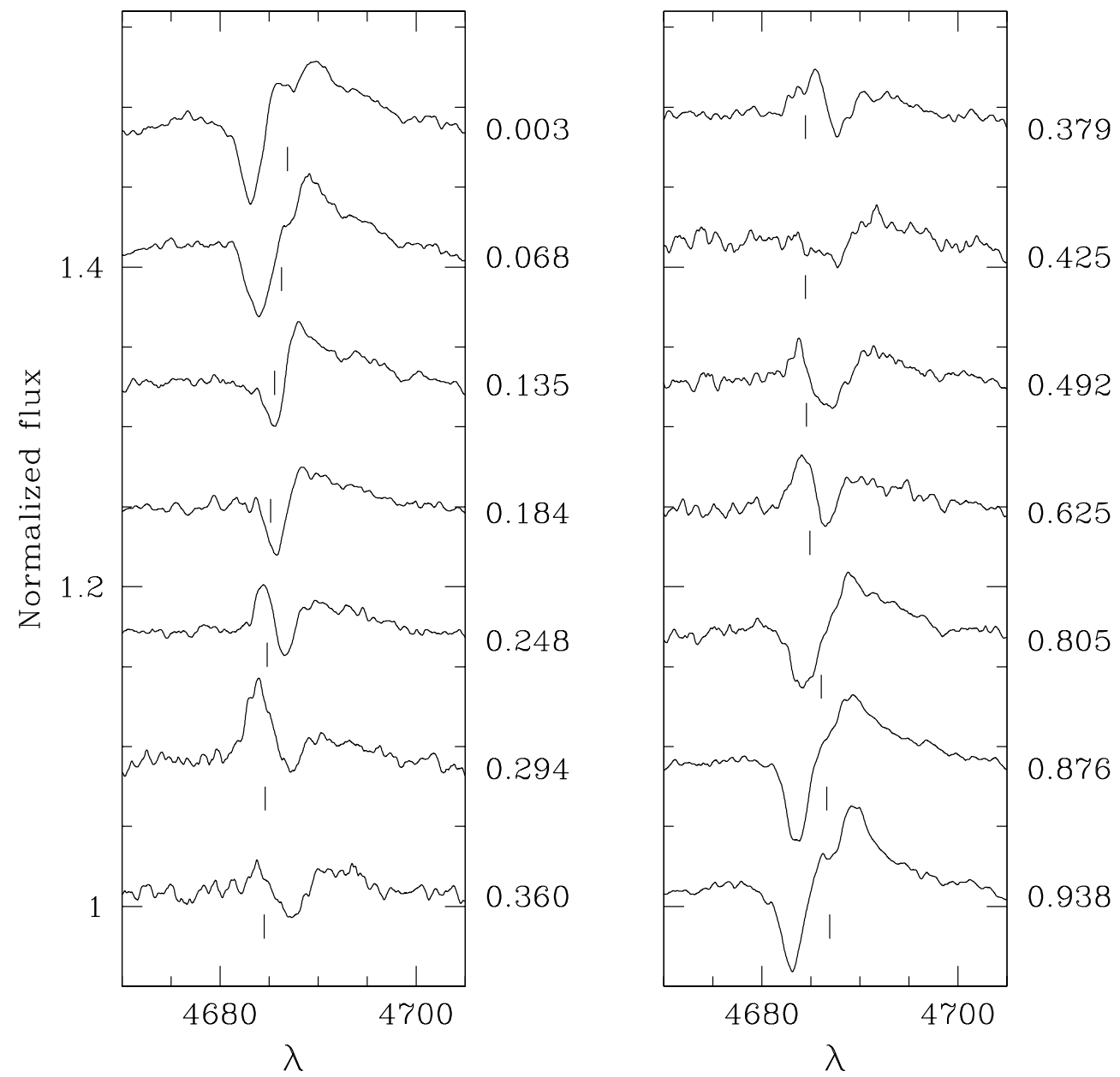

Fig. 5. Profile variations of the He II $\lambda 4686$ line in the spectrum of HD 93403 as a function of orbital phase. The labels indicate the orbital phases while the small ticks yield the position of the primary's line as derived from the orbital solution of Rauw et al. (2000). Note that the $R V \mathrm{~s}$ of the absorption component are in excellent agreement with the orbital motion of the secondary star.

separation, we would expect a variation of the colliding wind X-ray flux by a factor 1.6 between apastron and periastron (see Fig. 3). The fact that the observed modulation in the medium energy band is clearly lower than a factor 1.6 could indicate that a sizeable fraction of the X-ray emission from HD 93403 is intrinsic to the winds of the individual components or that radiative cooling affects the properties of the plasma to some extent (see also below). Other factors such as radiative braking (Gayley et al. 1997) could also play a role in the wind interaction.

For solar abundances the postshock temperature of the shocked material is expected to be $1.36 \times 10^{7} v^{2}(\mathrm{~K})$ where the preshock velocity is expressed in $1000 \mathrm{~km} \mathrm{~s}^{-1}$ (Stevens et al. 1992). As indicated by the wind momentum ratio $\mathcal{R}$ in Table 3 , the wind of the primary most probably dominates that of the secondary and the wind collision region should be wrapped around the secondary star. Since the shock occurs within the wind acceleration zones, the winds collide with velocities below $v_{\infty}$. If we assume a "standard" $\beta$-velocity law for the winds in HD 93403, we find that the postshock temperature should be about $40-50 \%$ higher at apastron than at periastron. Though the variations in the hard $(2.5-10.0 \mathrm{keV})$ band are clearly less significant than in the soft and medium bands, variability at these energies is expected to result from the combination of the global $1 / r$ modulation and the changes of the temperature of the emitting plasma.

Assuming a distance of $3.2 \mathrm{kpc}$ and correcting the observed flux for an interstellar column density of $0.37 \times$ $10^{22} \mathrm{~cm}^{-2}$, we obtain an average dereddened luminosity of $L_{\mathrm{X}}=3.7 \times 10^{33} \mathrm{erg} \mathrm{s}^{-1}$ in the $0.5-2.5 \mathrm{keV}$ range. Adopting the bolometric luminosity of the binary as derived by Rauw et al. (2000) yields $\log \left(L_{\mathrm{X}} / L_{\mathrm{bol}}\right)=-6.13$. This ratio is less extreme than the value $(-5.83)$ derived by Chlebowski \& Garmany (1991) from their EINSTEIN data. The larger ratio quoted by Chlebowski \& Garmany mainly reflects their value of $L_{\mathrm{X}}$ which is about 2.5 times larger than the luminosity we obtain from our EPIC spectra.

It is worth emphasizing that the HRI count rates of HD 93403 are in extremely good agreement with the luminosities and count rates inferred from our EPIC data. For instance, the parameters of the $\Phi=0.265$ EPIC spectra can be converted into a theoretical HRI count rate 
of $4.1 \times 10^{-2} \mathrm{cts} \mathrm{s}^{-1}$ in excellent agreement with the results of the ROSAT observations obtained three to eight years prior to the $X M M$ data. This seems to rule out a long term variation of the X-ray luminosity as the cause of the discrepancy between the XMM and EINSTEIN results. Part of the difference in $L_{\mathrm{X}}$ therefore most probably arises from the fact that Chlebowski \& Garmany (1991) used luminosities from Chlebowski et al. (1989) that assume an optically thin plasma with $k T=0.5 \mathrm{keV}$ and affected by interstellar absorption only.

The fact that our $\log \left(L_{\mathrm{X}} / L_{\mathrm{bol}}\right)$ value is not much larger than the "canonical" value for single stars provides further evidence that the X-ray emission is not totally due to colliding wind emission and hence single star emission is likely to play a significant role.

\section{The optical data}

\subsection{Emission lines}

The He II $\lambda 4686$ line consists of a mixture of emission and absorption features (see Fig. 5). Observations taken at the same orbital phase but during different observing runs, separated by several years, display the same overall morphology.

As pointed out by Rauw et al. (2002a), the radial velocities $(R V \mathrm{~s})$ of the absorption component closely matches the orbital motion of the secondary star. The He II $\lambda 4686$ line appears thus in absorption in the spectrum of the O7 secondary, lending further support to the main sequence luminosity class proposed for this star (Rauw et al. 2000). We notice that the strength of the secondary's absorption varies as a function of orbital phase. The absorption appears stronger around $\Phi \simeq 0.9$ and weaker near phase 0.4 . Some of these variations might be due to the blending with the emission component(s), but it seems unlikely that the whole phenomenon could be attributed to blending effects.

The emission component displays strong phase-locked variability. Between phases 0.805 and 0.135 , we observe a broad asymmetric emission with a prominent extended red wing. Between phases 0.248 and 0.625, the emission is blue-shifted and appears fainter, much narrower and more symmetric. Between $\Phi=0.360$ and 0.492 , the emission strength is considerably reduced and at some phases there is virtually no emission at all. The tickmarks in Fig. 5 indicate the $R V \mathrm{~s}$ of the primary component. We see that the $R V$ of the emission peak varies roughly in phase with the primary, though with a larger amplitude and a larger systemic velocity: the emission peak is clearly redshifted with respect to the primary between phases 0.805 and 0.184 and slightly blue-shifted at phases $0.248,0.294$, 0.492 and 0.625 . Some hints that the primary spectrum contributes a faint narrow emission to the He II $\lambda 4686$ line are found at $\Phi=0.876,0.938,0.003$ and 0.068 . This stellar emission may also contribute to the narrow line seen between $\Phi=0.248$ and 0.625 .

The $\mathrm{H} \alpha$ profile appears quite complex with a mixture of absorptions and emissions (see Fig. 6). The line seems to

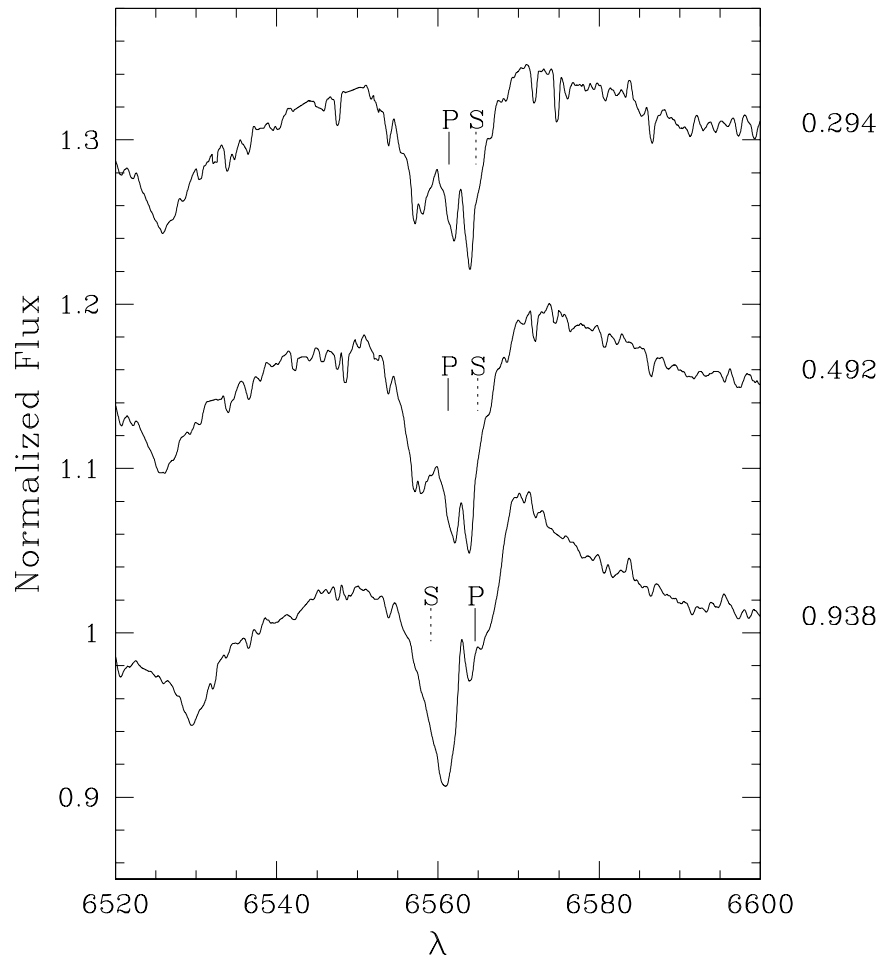

Fig. 6. Example of the line profiles of the $\mathrm{H} \alpha$ line in the spectrum of HD 93403. The $\mathrm{P}$ and $\mathrm{S}$ labels indicate the position of the lines of the primary and secondary respectively as expected from the orbital solution. Note the sharp nebular emission line at $6562.9 \AA$.

consist of an absorption belonging to the secondary and a blue-shifted absorption that is most probably associated with the primary. Underlying these features, there is a broad, roughly symmetric, emission line extending over about $70 \AA$. The profile is further polluted by a rather strong nebular $\mathrm{H} \alpha$ emission and by telluric water vapor absorptions. The intensity of both of these latter features depends on the weather conditions during the observations. In our normalized spectra, the relative intensity of the nebular emission increases when the atmospheric turbulence increases. In fact, a larger seeing means that the fiber of the FEROS instrument misses part of the stellar flux while the nebular photons that come from a diffuse extended region enter the fiber without significant losses.

Despite these complications, we find that the overall variations of the $\mathrm{H} \alpha$ profile are reminiscent of those of He II $\lambda 4686$. Figure 6 displays a red-shifted emission at $\Phi=0.938$, while there is no trace of such a feature at the other two phases shown in this plot. Conversely, the strength of the blue-shifted absorption appears reduced at phase 0.294 (i.e. when we observe a narrow, blue-shifted, emission peak in the He II $\lambda 4686$ line).

\subsection{Absorption lines}

In addition to the effects discussed hereabove, the spectrum of HD 93403 displays also variations of the relative strength of the absorption lines of the two stars. Whereas 

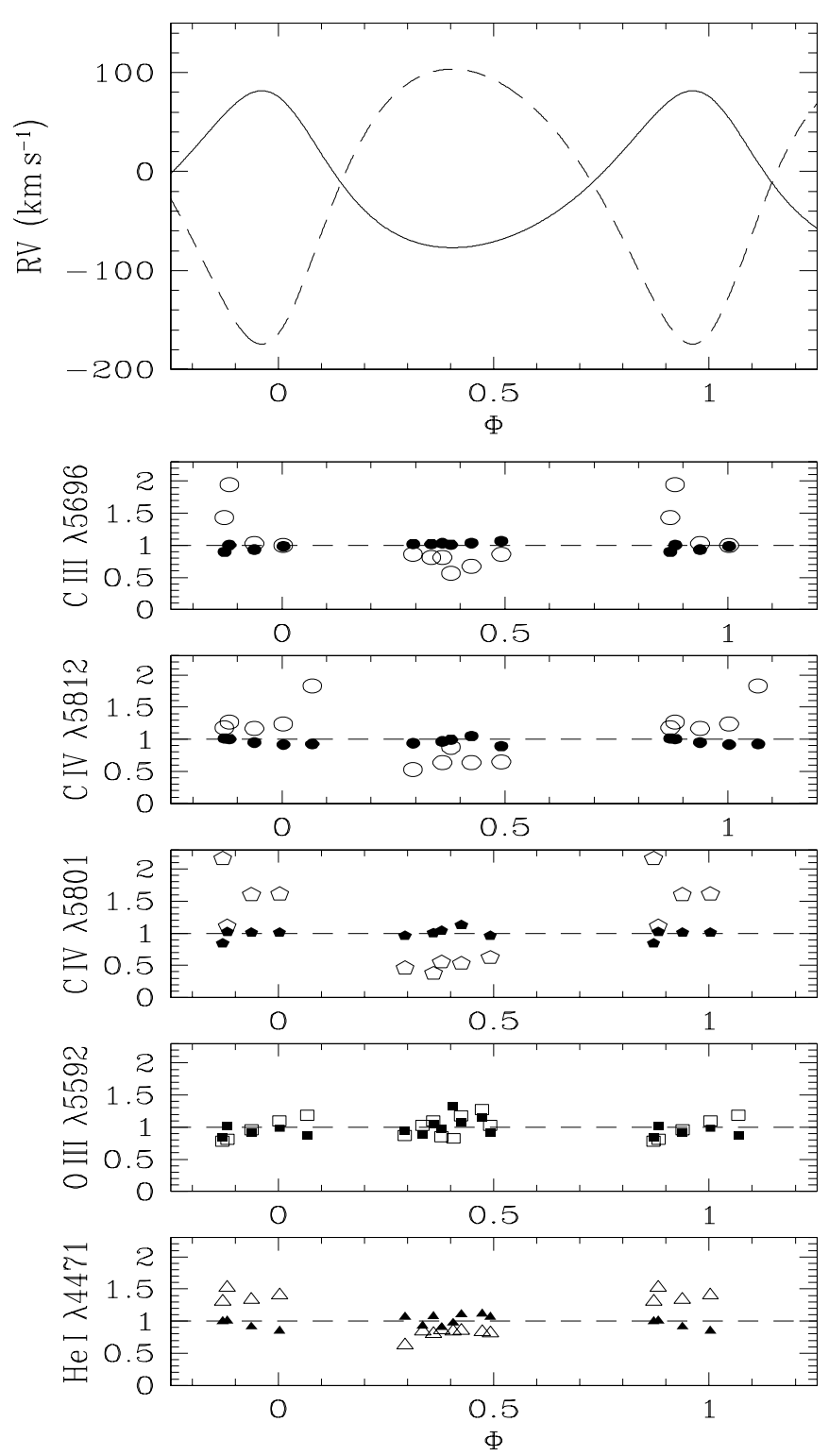

Fig. 7. Upper panel: orbital solution of HD 93403 as derived from the He I $\lambda 4471$ absorption line (Rauw et al. 2000). Phase 0.0 corresponds to periastron passage. The other panels display the ratio of the equivalent width of individual lines with respect to the mean $E W$ averaged over all available phases. Filled symbols stand for the primary's lines, whereas open symbols correspond to the secondary's lines.

the intensities of the primary's absorptions remain roughly constant over the orbital cycle, we observe substantial changes in the equivalent width $(E W)$ of the secondary's lines. Figure 7 displays the ratio between the individual equivalent widths of the different lines that could be deblended at a given orbital phase and the corresponding mean $E W$ averaged over all available phases. In general, the secondary's lines appear stronger at orbital phases when the secondary is moving towards $\mathrm{us}^{3}(\Phi \in[0.8,0.1])$. This is very similar to the "Struve-Sahade" effect (SSE)

3 This is very similar to what we observe for the absorption component of the He II $\lambda 4686$ blend.
Table 4. Properties of the absorption lines in the optical spectrum of the secondary star investigated for the Struve-Sahade effect. I.P. stands for the ionization potential of the species corresponding to one ionizing stage below that of the relevant ions. E.P. yields the excitation potential of the lower level of the transition.

\begin{tabular}{lrcc}
\hline \hline Line & $\begin{array}{c}\text { I.P. } \\
(\mathrm{eV})\end{array}$ & $\begin{array}{c}\text { E.P. } \\
(\mathrm{eV})\end{array}$ & SSE \\
\hline He I $\lambda 4471$ & 0.0 & 21.0 & Yes \\
O III $\lambda 5592$ & 35.1 & 33.8 & No \\
C III $\lambda 5696$ & 24.4 & 32.1 & Yes \\
C IV $\lambda 5801$ & 47.9 & 37.5 & Yes \\
C IV $\lambda 5812$ & 47.9 & 37.5 & Yes \\
\hline
\end{tabular}

described for instance by Stickland (1997) and Bagnuolo et al. (1999).

The Struve-Sahade effect in the optical spectrum of HD 93403 appears most prominent for the C IV $\lambda \lambda 5801$, 5812 lines whereas it is slightly less pronounced for He I $\lambda 4471$ and essentially absent in O III $\lambda 5592$. This latter result is somewhat surprising since the $\mathrm{C}$ IV doublet and the $\mathrm{O}$ III line have all a rather high excitation potential and involve ions that require large ionization potentials (see Table 4). These lines are thus most probably all formed quite deep within the photospheric layers. Another remarkable feature is the fact that the C III $\lambda 5696 \mathrm{emis}$ sion line also displays a moderate SSE.

\subsection{Discussion}

The variability of the He II $\lambda 4686$ line indicates a dependence of the intensity of the broader emission on the orbital separation; the emission is strongest around phases 0.80-0.15 and vanishes when the stars are heading towards apastron. A possible explanation could be that the broader emission component is generated in the interaction between the two stars and scales roughly with some power of $1 / r$.

These variations could reflect a change of the primary's mass loss rate due to some sort of tidal interaction. The fact that the emission has a larger $R V$ amplitude $\left(\sim 180 \mathrm{~km} \mathrm{~s}^{-1}\right)$ than the orbital motion of the primary $\left(79 \mathrm{~km} \mathrm{~s}^{-1}\right)$ could imply that this emission arises in the tidal bulge on the side of the primary not facing the secondary. Indeed, in order to follow the orbital motion, the material in this part of the wind would have to move at larger velocities than the center of mass of the primary. The lack of significant emission from the bulge on the side facing the secondary could be due to the X-ray emission from a wind collision. These X-rays could ionize this part of the wind and hence prevent the formation of the He II $\lambda 4686$ emission line. Since the X-ray luminosity is largest around periastron, it should have the largest ionizing power at this phase. Nevertheless, it is around periastron that the He II $\lambda 4686$ emission appears strongest. This could indicate that the emission comes from the "rear" 
side of the primary with an intensity that scales as some power of $1 / r$ reflecting the dependence of the tidal interaction on the orbital separation.

There are however a couple of problems with this explanation. For instance, it is surprising that our data reveal a stronger emission at phases prior to periastron passage (e.g. $\Phi=0.876$ ) than at the symmetric phases after periastron passage (e.g. $\Phi=0.135$ ). Simulations of the tidal interactions in an eccentric massive binary (Moreno \& Koenigsberger 1999) yield an asymmetrical "time-profile" of the amplitude of the maximum radial displacement of the surface layers of the primary star: the tidal bulge is on average smaller prior to periastron than afterwards. Though the model parameters used by Moreno \& Koenigsberger were adjusted to match the $\iota$ Ori binary $(e=0.764)$ and although it is not clear how the wind emission lines react to a tidal bulge, it seems likely that the emission should be on average stronger after periastron also in HD 93403, which is not what we observe. Another comment concerns the Roche lobe radii listed in Table 3. For reasonable values of the orbital inclination, the primary's Roche lobe radius at periastron is significantly larger than the "typical" radius of an O 5.5 supergiant $\left(\sim 24 R_{\odot}\right)$ and it seems thus unlikely that a strong tidal interaction could take place in HD 93403.

Alternatively, the broad He II $\lambda 4686$ emission could arise in the high density parts of a colliding wind interaction. Let us recall that radiative cooling is expected to be most efficient around periastron and it is therefore around these phases that the optical line emission (arising from relatively cool material) should reach its maximum. The easiest way to account for most of the properties of the broad emission component is to assume that the excess emission is coming from the trailing arm of the shock. Stevens \& Howarth (1999) pointed out that the trailing edge of the shock region should produce more line emission because of the greater density at this site. Due to the Coriolis deflection, the shock zone in a wind interaction is expected to be strongly curved. If this effect is strong enough, then the trailing arm of the shock could be roughly perpendicular to the binary axis (see our sketch in Fig. 4) and we would expect a redshifted emission between phases 0.8 and 0.13 which is pretty much what we observe. It is worth pointing out that our observations of the He II $\lambda 4686$ line display indeed qualitative similarities to the synthetic line profile variations simulated by Stevens \& Howarth (1999). We caution however that a very strong bending of the shock is required to explain the red wing that we observe at $\Phi=0.184$. Without a strong bending, the redward extension of the emission should already be considerably reduced at this phase.

Though the Struve-Sahade effect was already reported by Struve in the late 1930's (Struve 1937), its origin is still not established and different binaries sometimes lead to contradictory pictures (see e.g. Bagnuolo et al. 1999). Sahade (1959) proposed that a gas stream from the primary that sweeps past the trailing side of the secondary could cause an obscuration of the secondary at orbital phases when it is receding. Alternatively, Abhyankar (1959) suggested that the lines could strengthen during approaching phases due to an enhanced density on the leading hemisphere of the secondary. Gies et al. (1997) proposed that the SSE could result from photospheric heating by the X-rays emitted by a colliding wind interaction. Due to the Coriolis force, the bow shock is deflected in such a way that it would preferentially heat the leading hemisphere which is best seen during orbital phases of secondary approach. More recently, Gayley (2002) suggested that the SSE could result from external irradiation of the stellar surface that induces a transverse radiative force and hence transverse supersonic flows in the secondary's atmosphere. These transverse flows could break the receding/approaching symmetry of the secondary velocity structure and lead to shallower and broader absorption lines at phases when the secondary is receding.

Our observations of HD 93403 indicate that lines corresponding to highly excited species do not necessarily display the same behaviour. An obscuration effect of the secondary by the trailing arm of the wind collision zone can therefore be ruled out as the cause of the SSE in HD 93403. Such an effect should indeed affect all the lines of the secondary in the same way, which is not the case.

Let us remind what is known about the formation of the lines investigated here. The $E W$ of the He I $\lambda 4471$ line in O-star spectra is rather well correlated with the spectral type and hence the effective temperature though there is also a moderate dependence on gravity (see e.g. Voels et al. 1989 and references therein). On the other hand, the $E W$ s of the $\mathrm{C}$ IV $\lambda \lambda 5801,5812$ and $\mathrm{O}$ III $\lambda 5592$ lines in O-star spectra do not reveal any clear-cut dependence neither on the spectral type nor on the luminosity (Conti 1974). Finally, the strength of the C III $\lambda 5696$ emission is known to increase with luminosity (Walborn 1980). This emission most probably forms in the stellar photosphere; Cardona-Núñez (1978) showed that the upper level $3 \mathrm{~d}^{1} \mathrm{D}$ of the $\lambda 5696$ transition is overpopulated with respect to the lower level $3 \mathrm{p}^{1} \mathrm{P}^{0}$ through a combination of direct and dielectronic recombination processes and cascades from upper states.

The lack of variations of the primary's $E W \mathrm{~s}$ suggests that there are no significant variations of the dilution of the primary's lines and thus that the secondary's continuum flux remains constant. It seems unlikely that an increase of the local surface temperature could be the sole effect controlling the SSE in HD 93403. In fact, a local heating of the secondary's surface by the wind interaction zone, without a notable brightening of the secondary, is expected to reduce the $E W$ of the He I $\lambda 4471$ line when the secondary is moving towards us, which is the opposite of what we observe. Therefore, the SSE in HD 93403 must be caused by some perturbation of the physical conditions in the atmosphere of the secondary that affects the formation processes of some (but not all) lines but does not alter the overall brightness of the secondary. 


\section{Final remarks and conclusion}

We have presented observations of HD 93403 that reveal phase-locked variability of the X-ray flux and of the optical spectrum of this massive binary system. The colliding wind scenario offers a promising explanation of this variability. The bulk of the medium band X-ray emission probably arises in the wind interaction region near the point of head-on collision. The shocked wind material in this region is probably too hot to emit substantial optical emission and the cooling is essentially adiabatic. On the other hand, the optical line emission most probably forms in the trailing arm of the shock region where the density is higher and the plasma cools more efficiently through radiative cooling. It seems therefore that the $\mathrm{X}$-ray and optical data probe two distinct regions of the same interaction phenomenon.

An interesting question is whether the ram pressure of the secondary's wind is sufficient to hold off the primary's wind. In fact, if the primary's wind crushed on the secondary's surface, it would strongly perturb the local physical conditions (e.g. temperature, density, velocity field...) in the secondary's atmosphere. For a wind momentum ratio of $\mathcal{R}=3.33$ (Table 3 ) and assuming a standard velocity law with $\beta=1$ for both stars, we find that the shock should remain detached from the surface of the secondary star over the entire orbit. Although the figures in Table 3 provide only a crude estimate of the stellar parameters, we note that the shock should remain detached for values of $\mathcal{R}$ as large as 4.5. Therefore it seems unlikely that a direct impact of the primary's wind on the secondary's surface could be responsible for the Struve-Sahade effect.

Our results suggest some tracks for future investigations of the wind interactions in O-type binaries. Comparing the XMM-Newton spectra of HD 93403 and other early-type binaries, spanning a wide range of spectral types and orbital parameters, with detailed numerical simulations (e.g. Pittard 2000) should enable us to constrain the hydrodynamics of stellar winds and to investigate the effects of radiative braking and thermal conduction on the wind collision. Concerning the Struve-Sahade effect, it will be important to gather optical echelle spectroscopy of a sample of binaries, allowing to investigate a number of lines individually at high spectral resolution and with a high $S / N$ ratio. From a theoretical point of view, quantitative predictions of the behaviour of these lines under the different scenarios (particularly the Gayley 2002 scenario) are needed to allow a comparison with observations and hence to solve this long standing enigma.

Acknowledgements. The authors wish to thank the XMM-SOC team for their efforts to schedule the pointings at the right orbital phases and for their assistance in using the SAS software. Our thanks go also to Alain Detal for his help in installing the SAS, to Dr. Herman Hensberge for advising us in the reduction of the FEROS data and to Yaël Nazé for performing wind opacity calculations upon our request. We are greatly indebted to the Fonds National de la Recherche Scientifique (Belgium) for multiple assistance. This research is also supported in part by contract P4/05 "Pôle d'Attraction Interuniversitaire" (SSTCBelgium) and through the PRODEX XMM-OM and Integral Projects.

\section{References}

Abhyankar, K. D. 1959, ApJS, 4, 157

Bagnuolo, W. G. Jr., Gies, D. R., Riddle, R., \& Penny, L. R. 1999, ApJ, 527, 353

Cardona-Núñez, O. 1978, Ph.D. Thesis, University of Colorado, Boulder

Chen, W., \& White, R. L. 1991, ApJ, 366, 512

Chlebowski, T., Harnden, F. R. Jr., \& Sciortino, S. 1989, ApJ, 341,427

Chlebowski, T., \& Garmany, C. D. 1991, ApJ, 368, 241

Conti, P. S. 1974, ApJ, 187, 539

Corcoran, M. F. 1996, Rev. Mex. Astron. Astrofis. Conf. Ser., 5,54

Eichler, D., \& Usov, V. 1993, ApJ, 402, 271

Gayley, K. G. 2002, in Interacting Winds from Massive Stars, ed. A. F. J. Moffat, \& N. St-Louis, ASP Conf. Ser., 260, 583

Gayley, K. G., Owocki, S. P., \& Cranmer, S. R. 1997, ApJ, 475,786

Gies, D. R., Bagnuolo, W. G. Jr., \& Penny, L. R. 1997, ApJ, 479,408

Howarth, I. D., Siebert, K. W., Hussain, G. A. J., \& Prinja, R. K. 1997, MNRAS, 284, 265

Jansen, F., Lumb, D., Altieri, B., et al. 2001, A\&A, 365, L1

Kaastra, J. S. 1992, An X-ray spectral code for optically thin plasmas, Internal SRON-Leiden Report

Kahn, S. M., Leutenegger, M. A., Cottam, J., et al. 2001, A\&A, 365, L312

Kaufer, A., Stahl, O., Tubbesing, S., et al. 1999, The Messenger, 95, 8

Lamers, H. J. G. L. M., \& Leitherer, C. 1993, ApJ, 412, 771

Marchenko, S. V., Rauw, G., Antokhina, E. A., et al. 2000, MNRAS, 317, 333

Mason, K. O., Breeveld, A., Much, R., et al. 2001, A\&A, 365, L36

Mewe, R., Gronenschild, E. H. B. M., \& van den Oord, G. H. J. 1985, A\&AS, 62, 197

Moreno, E., \& Koenigsberger, G. 1999, Rev. Mex. Astron. Astrofis., 35, 157

Pittard, J. M. 2000, Ph.D. Thesis, University of Birmingham Pittard, J. M., \& Stevens, I. R. 1997, MNRAS, 292, 298

Pittard, J. M., Stevens, I. R., Corcoran, M. F., et al. 2000, MNRAS, 319, 137

Rauw, G., Vreux, J.-M., \& Bohannan, B. 1999, ApJ, 517, 416

Rauw, G., Sana, H., Gosset, E., et al. 2000, A\&A, 360, 1003

Rauw, G., Nazé, Y., Carrier, F., et al. 2001, A\&A, 368, 212

Rauw, G., Sana, H., Vreux, J.-M., Gosset, E., \& Stevens, I. R. 2002a, in Interacting Winds from Massive Stars, ed. A. F. J. Moffat, \& N. St-Louis, ASP Conf. Ser., 260, 449

Rauw, G., Vreux, J.-M., Antokhin, I., et al. 2002b, in New Visions of the X-ray Universe in the XMM-Newton and Chandra Era, ed. F. Jansen, ESA SP-488, in press

Sahade, J. 1959, PASP, 71, 151

Sana, H., Rauw, G., \& Gosset, E. 2001, A\&A, 370, 121

Stevens, I. R., Blondin, J. M., \& Pollock, A. M. T. 1992, ApJ, 386,265 
Stevens, I. R., \& Howarth, I. D. 1999, MNRAS, 302, 549

Stickland, D. J. 1997, The Observatory, 117, 37

Strüder, L., Briel, U., Dennerl, K., et al. 2001, A\&A, 365, L18

Struve, O. 1937, ApJ, 85, 41

Thaller, M. L., Gies, D. R., Fullerton, A. W., Kaper, L., \& Wiemker, R. 2001, ApJ, 554, 1070

Turner, M. J. L., Abbey, A., Arnaud, M., et al. 2001, A\&A, $365, \mathrm{~L} 27$ van der Hucht, K. A. 2002, in Multifrequency Behaviour of High Energy Cosmic Sources, ed. F. Giovanelli, \& L. SabauGraziati, Mem. Soc. Astro. Italia, in press

Voels, S. A., Bohannan, B., Abbott, D. C., \& Hummer, D. G. 1989, ApJ, 340, 1073

Walborn, N. R. 1980, ApJS, 44, 535

Waldron, W. L., Corcoran, M. F., Drake, S. A., \& Smale, A. P. 1998, ApJS, 118, 217 